\section{Itinerários terapêuticos de mulheres com morbidade materna grave}

\section{Treatment itineraries of women with severe maternal morbidity}

\author{
Itinerarios terapéuticos de mujeres con \\ morbilidad materna grave
}

Magna Santos Andrade 1

Elisabeth Meloni Vieira 2

doi: 10.1590/0102-311X00091917

\section{Resumo}

Este estudo objetivou identificar os itinerários terapêuticos de mulheres acometidas por morbidade materna grave. Trata-se de pesquisa qualitativa, descritiva e exploratória realizada em um hospital universitário de referência em gestação de alto risco do interior do Estado de São Paulo, Brasil. Participaram 16 mulheres com complicações graves na gestação, parto ou pós-parto. Os dados foram coletados por entrevistas semiestruturadas, gravadas, transcritas e analisadas mediante Análise de Conteúdo. Os resultados mostram que as mulheres, ao iniciarem o processo de adoecimento, seguem trajetórias diversas, buscando serviços da baixa e alta complexidade, procurando também por vizinha, farmácia e uso de automedicação. Destacam-se entraves referentes ao acolhimento, continuidade do cuidado, resolutividade e referência na rede de atenção à saúde. Observaram-se importantes pontos críticos em relação à assistência obstétrica, sendo a peregrinação por serviços de saúde, a demora do encaminhamento e a violência institucional, realidades vivenciadas pelas mulheres. O serviço terciário foi apontado como acolhedor e eficaz no atendimento. Este estudo permitiu a identificação dos problemas que existem em toda a cadeia de cuidados obstétricos, e tal conhecimento permite o entendimento de como o sistema de saúde se organiza em relação ao atendimento das mulheres com complicações maternas graves.

Morbidade; Saúde da Mulher; Assistência Integral à Saúde
Correspondência

M. S. Andrade

Rod. Lomanto Junior, BR 407, km 127, Senhor do Bonfim, BA 48970-000, Brasil.

magnaenf@yahoo.com.br

1 Universidade do Estado da Bahia, Senhor do Bonfim, Brasil.

2 Universidade de São Paulo, Ribeirão Preto, Brasil. 


\section{Introdução}

No ano 2000, a Organização das Nações Unidas estabeleceu oito propostas denominadas Objetivos de Desenvolvimento do Milênio (ODM) dos quais o quinto objetivo é melhorar a saúde materna. Para isso, foram elaboradas duas metas: o acesso universal à saúde reprodutiva e a redução da razão de mortalidade materna em três quartos de 1990 até 2015 1,2. A despeito de diferenças segundo regiões no mundo, observou-se decréscimo das razões de mortalidade materna, entretanto o ritmo de desaceleração não foi suficiente para se cumprir essa meta, alcançada por nove países, mas não o Brasil 3 .

A morte materna permanece um grave problema de saúde pública e representa violação aos direitos humanos, pois $98 \%$ dos casos são evitáveis 4 . A investigação dos casos produz informações importantes sobre o perfil dos óbitos, orientando medidas para seu enfrentamento. Porém, as investigações são limitadas, face ao pequeno número absoluto de mortes e às dificuldades devido ao sub-registro desses óbitos nos sistemas oficiais de mortalidade, além da má qualidade da informação nos serviços de saúde.

Sendo assim, o estudo da morbidade materna grave se mostra promissor na produção de conhecimento sobre os fatores que podem contribuir para o óbito materno e em relação à assistência obstétrica prestada. Os casos de morbidade materna grave são mais frequentes do que os óbitos. A sobrevivência das mulheres viabiliza a coleta de informações diretamente com as usuárias, por entrevistas, superando problemas referentes à inconsistência das informações nos serviços de saúde 5,6.

Entende-se por mulheres com morbidade materna grave aquelas que possuem condições potencialmente ameaçadoras de vida, que são graves complicações que ocorrem na gestação, parto e até 42 dias de pós-parto e aqueles casos de extrema gravidade (quase morte) nesse mesmo período são denominados near miss materno 7 .

As mulheres com complicações maternas graves recebem diversos cuidados e tratamentos, calcados em saberes profissionais e populares, para solucionar os seus problemas. Nessa perspectiva, é relevante compreender o caminho percorrido pelas usuárias na busca de atenção à saúde, conhecer os dispositivos e movimentos utilizados pela mulher e familiares no enfrentamento da doença, sendo essa trajetória conhecida como itinerário terapêutico 8 .

Estudo realizado na década de 1990 analisou a trajetória das mulheres com complicações obstétricas segundo três momentos (decisão pelo cuidado, acesso aos serviços e qualidade da assistência) e identificou que além dos aspectos que envolvem as usuárias (características socioeconômicas e culturais) e os serviços de saúde (qualificação da equipe, estrutura física etc.), há fatores que também influenciam no acesso à assistência: transporte disponível, condições de estradas, custos de deslocamento e distância, no entanto o estudo revelou que os atrasos para o cuidado adequado devem-se especialmente às dificuldades nas instituições de saúde (profissionais despreparados, materiais e equipamentos insuficientes e referência ineficiente) 9 .

Estudos que abordam a experiência de mulheres com graves complicações maternas ainda são poucos, e o comportamento dessas usuárias na busca por cuidados de saúde é raramente documentado 10 .

Pesquisas sobre morbidade materna grave se concentram particularmente nos indicadores e fatores associados às complicações 11,12. Estudos qualitativos mostram que o itinerário terapêutico das mulheres com morbidade materna grave não gira em torno apenas de aspectos biomédicos, mas também possui relação com as desigualdades de gênero, de educação, de renda, de cultura, de distribuição dos serviços de saúde, além das dificuldades de atendimento na rede assistencial 13,14.

Diante do exposto, o presente estudo teve como objetivo identificar os itinerários terapêuticos de mulheres acometidas por morbidade materna grave residentes no interior do Estado de São Paulo, Brasil.

\section{Método}

Pesquisa qualitativa, norteada pelo referencial teórico do modelo de sistemas de cuidado à saúde proposto por Kleinman ${ }^{15}$, que concebe que a saúde, a doença e os aspectos relacionados ao cuidado estão 
articulados como um sistema cultural que envolve o simbólico (constituído por valores, significados e normas de comportamento), o religioso e o familiar.

Para esse autor, o sistema de cuidado à saúde é constituído pela interação de três subsistemas: profissional, popular e folk 15 .

O subsistema profissional é formado pelos profissionais de saúde que detêm o conhecimento técnico-científico, constituído pela medicina tradicional ocidental e pelas demais medicinas reconhecidas, a exemplo da chinesa 15 .

No subsistema popular, o adoecimento é inicialmente reconhecido e tratado, sendo em geral o ponto de partida do processo terapêutico, e envolve as noções do senso comum, compartilhadas pelos indivíduos, suas famílias, suas redes sociais e membros próximos da comunidade 15.

O subsistema folk envolve as crenças e culturas em que o indivíduo está inserido, e as doenças são identificadas e tratadas na perspectiva de tais valores. Nesse contexto, destacam-se os curandeiros, as rezadeiras, os pastores, os pais de santos dentre outros que personificam as crenças e valores dos grupos sociais 15 .

No Brasil, a relação entre os itinerários terapêuticos e a situação de pobreza é bem próxima, principalmente pela extrema desigualdade social e diversidade cultural que marcam o país, o que reflete diretamente na busca por cuidado 16 . Por isso, o estudo sobre itinerários terapêuticos deve envolver não apenas a utilização e disponibilidade dos serviços de saúde, mas também é necessário considerar o contexto sociocultural em que o adoecimento se desenvolve, analisando os aspectos individuais, coletivos e a relação entre cultura, sociedade e condutas adotadas ${ }^{17}$. Para tanto, o estudo dos itinerários terapêuticos com base nos subsistemas de saúde propostos por Kleinman 15 é fundamental, pois viabiliza o entendimento do processo assistencial em sua macro e microestrutura.

Para coleta de dados, adotou-se a entrevista semiestruturada, realizada com um roteiro pré-estabelecido constituído por questões previamente elaboradas 18. A entrevista semiestruturada foi avaliada como mais adequada para este estudo por obter informações de mulheres hospitalizadas, não sendo viável, por exemplo, a etnografia ou observação participante, em razão da impossibilidade de saber qual mulher desenvolveria morbidade materna grave, onde e quando isso aconteceria.

Foram entrevistadas 16 mulheres no pós-parto, com morbidade materna grave, especialmente casos de condições potencialmente ameaçadoras de vida porque aquelas que desenvolveram near miss materno podem não se lembrar detalhadamente dos fatos pela gravidade do quadro. As entrevistas ocorreram em um hospital universitário de referência em gestação de alto risco do interior do Estado de São Paulo. Para a classificação das mulheres enquanto condições potencialmente ameaçadoras de vida, utilizaram-se os critérios da Organização Mundial da Saúde (OMS) 7. Todavia, esse quadro de classificação é bastante heterogêneo, havendo casos mais ou menos graves, o que de algum modo afeta o itinerários terapêuticos na busca por cuidados.

Os critérios de inclusão dos casos foram: mulheres internadas no hospital universitário e que estavam no puerpério (até 42 dias de pós-parto); com idade a partir de 18 anos; apresentaram episódio de morbidade materna grave (condições potencialemnte ameaçadoras de vida) de acordo com a classificação da OMS 7; estavam no momento da entrevista em boas condições de saúde, aptas a fornecer informações.

As entrevistas ocorreram entre agosto de 2015 a fevereiro de 2016, e a coleta de dados foi encerrada após a saturação dos relatos.

Para a análise dos dados, utilizou-se a Análise de Conteúdo e, para isso, foram seguidos os seguintes procedimentos metodológicos: categorização, inferência, descrição e interpretação. Os dados foram inicialmente transcritos; em seguida, realizou-se leitura compreensiva do material, decomposição do material em partes, distribuição das partes em categorias, realização de inferências dos resultados e interpretação dos dados pela fundamentação teórica adotada 19 .

Foram construídas quatro categorias fundamentadas no roteiro de entrevista e análise das narrativas: decisão e busca por atendimento diante da percepção da complicação (sinais e sintomas iniciais, primeira pessoa ou o primeiro local procurado no momento do adoecimento, tempo para busca do serviço de saúde e quem foi o responsável pela iniciativa, uso de automedicação), dificuldades de acesso aos serviços de saúde (local de realização do pré-natal, recebimento de tratamento na complicação, quantidade e tipo de serviços de saúde procurados até a efetivação do cuidado, encaminhamentos realizados a partir do primeiro serviço), qualidade da assistência (detalhamento de cada atendimento, 
dificuldades encontradas nos serviços e aquilo que poderia ter sido diferente na assistência recebida) e perda de seguimento de casos de morbidade materna grave (categoria emergente que surgiu do relato de uma das entrevistadas).

As perguntas utilizadas no roteiro de entrevista foram elaboradas de modo a compreender o itinerário terapêutico das mulheres pelos subsistemas de saúde propostos por Kleinmam 15.

Este estudo faz parte de uma pesquisa maior denominada Pré-natal no Celular (PRENACEL), que verificou se uma estratégia de intervenção poderia impactar em melhor atenção pré-natal e um dos recortes analisou os casos de morbidade materna grave. O PRENACEL coletou dados de mulheres no pós-parto imediato, por isso não foi pesquisado o itinerário terapêutico no puerpério.

O estudo foi aprovado pelo Comitê de Ética em Pesquisa do Centro de Saúde Escola, Faculdade de Medicina de Ribeirão Preto, Universidade de São Paulo (parecer no 971.458). As participantes assinaram Termo de Consentimento Livre e Esclarecido.

\section{Resultados e discussão}

As mulheres possuíam entre 18 e 42 anos, eram pretas ou pardas, viviam com companheiro (casada/ união estável), tinham oito anos ou mais de escolaridade, pertenciam à classe social C (Critério de Classificação Econômica Brasil) 20, todas fizeram o pré-natal, metade iniciou no primeiro trimestre e metade fez menos de seis consultas, todas iniciaram o pré-natal na atenção básica, e dentre elas um terço continuou o acompanhamento pré-natal no hospital de referência (Tabela 1).

Pela análise dos relatos, construíram-se quatro categorias relacionadas ao IT, descritas abaixo.

\section{Decisão e busca por atendimento diante da percepção da complicação}

Foram encontrados diferentes comportamentos das mulheres ao apresentar os primeiros sintomas. A maioria das entrevistadas procurou o serviço de saúde assim que adoeceu, destacando-se o início do itinerário terapêutico pelo subsistema profissional.

Inicialmente, os sintomas apresentados mais relatados pelas mulheres foram cefaleia e dor abdominal, também foram referidos tontura, alterações visuais, sangramento vaginal, vômito, edema e dispneia. Observou-se sintomatologia semelhante em mulheres com complicações maternas pesquisadas em Fernandópolis, São Paulo 21.

O modo como se instala e a percepção da usuária sobre o adoecimento, o reconhecimento da mulher e familiares sobre a gravidade do quadro, aspectos culturais e disponibilidade de recursos financeiros influenciam no comportamento referente à busca por serviços de saúde, por isso o caminho percorrido pelas usuárias dentro das instituições de saúde é apenas parte do enfrentamento da complicação em uma dinâmica muito mais complexa 10,22.

$\mathrm{Na}$ busca de assistência, o indivíduo encontra diferentes maneiras para a resolução dos seus problemas de saúde. As ações de cuidados à saúde consistem em respostas organizadas em aspectos socioculturais direcionados para o enfrentamento da doença, e para isso há interação entre os setores popular e profissional 23. Nesse aspecto, as disponibilidades circunstanciais também são fatores relevantes para a tomada de decisão 16.

“...eu chamei minha vizinha que é enfermeira, ela mediu minha pressão e viu que a minha pressão tava muito alta..." (Entrevistada 13).

"No sábado, eu descobri que eu não me senti bem aí o cara foi medir a pressão na farmácia, aí o rapaz me mandou para o hospital ai eu peguei e fui..." (Entrevistada 1).

A maioria das mulheres entrevistadas procurou o serviço assim que adoeceu, mas em poucos casos se verificou a permanência em casa por horas ou dias desde o surgimento dos sintomas até a busca por assistência qualificada.

"Foi no sábado que eu senti muita dor forte, aí comecei a sentir no sábado, aí quando foi na segunda eu já procurei o médico, pra mim ver o que que era" (Entrevistada 9).

Pesquisa em hospitais brasileiros mostrou que 5,3\% das mulheres com morbidade materna grave ou que morreram demoraram a procurar pelo serviço de saúde no momento do adoecimento e houve associação positiva entre o atraso na assistência e a gravidade do desfecho materno 24 . 
Tabela 1

Descrição das mulheres portadoras de morbidade materna grave.

\begin{tabular}{|c|c|c|c|c|c|c|c|c|}
\hline & $\begin{array}{l}\text { Idade } \\
\text { (anos) }\end{array}$ & $\begin{array}{l}\text { Situação } \\
\text { conjugal }\end{array}$ & $\begin{array}{c}\text { Anos de } \\
\text { escolaridade }\end{array}$ & $\begin{array}{l}\text { Classe } \\
\text { social * }\end{array}$ & Raça/Cor & $\begin{array}{l}\text { Número de } \\
\text { consultas de } \\
\text { pré-natal }\end{array}$ & $\begin{array}{l}\text { Número } \\
\text { de partos } \\
\text { anteriores }\end{array}$ & $\begin{array}{c}\text { Tipo de morbidade } \\
\text { materna grave } \\
\text { apresentada }\end{array}$ \\
\hline Entrevistada 1 & 31 & Casada & 06 & C & Parda & 8 & 4 & $\begin{array}{c}\text { Pré-eclampsia grave/ } \\
\text { uso de sulfato de } \\
\text { magnésio }\end{array}$ \\
\hline Entrevistada 2 & 29 & Solteira & 12 & C & Branca & 7 & 0 & Hipertensão grave \\
\hline Entrevistada 3 & 24 & $\begin{array}{l}\text { Mora } \\
\text { junto }\end{array}$ & 08 & D & Parda & 6 & 1 & $\begin{array}{l}\text { Síndrome HELLP/ } \\
\text { pré-eclampsia grave/ } \\
\text { plaquetopenia/uso de } \\
\text { sulfato de magnésio }\end{array}$ \\
\hline Entrevistada 4 & 37 & Casada & 4 & C & Branca & 5 & 5 & $\begin{array}{l}\text { Descolamento } \\
\text { prematuro de } \\
\text { placenta }\end{array}$ \\
\hline Entrevistada 5 & 29 & Casada & 16 & B & Branca & 9 & 2 & Síndome HELLP \\
\hline Entrevistada 6 & 40 & Solteira & 8 & C & Parda & 7 & 2 & Pré-eclampsia grave \\
\hline Entrevistada 7 & 42 & $\begin{array}{l}\text { Mora } \\
\text { junto }\end{array}$ & 1 & D & Parda & 7 & 2 & $\begin{array}{l}\text { Descolamento } \\
\text { prematuro de } \\
\text { placenta }\end{array}$ \\
\hline Entrevistada 8 & 40 & $\begin{array}{l}\text { Mora } \\
\text { junto }\end{array}$ & 12 & C & Branca & 4 & 0 & $\begin{array}{l}\text { Pré-eclampsia grave/ } \\
\text { síndrome HELLP }\end{array}$ \\
\hline Entrevistada 9 & 24 & Solteira & 12 & C & Parda & 8 & 2 & $\begin{array}{c}\text { Retorno ao centro } \\
\text { cirúrgico }\end{array}$ \\
\hline Entrevistada 10 & 18 & Casada & 12 & C & Branca & 10 & 0 & $\begin{array}{c}\text { Pré-eclampsia grave/ } \\
\text { uso de sulfato de } \\
\text { magnésio }\end{array}$ \\
\hline Entrevistada 11 & 21 & $\begin{array}{l}\text { Mora } \\
\text { junto }\end{array}$ & 11 & C & Branca & $\begin{array}{c}\text { Sem } \\
\text { informação }\end{array}$ & 0 & Endometrite \\
\hline Entrevistada 12 & 37 & $\begin{array}{l}\text { Mora } \\
\text { junto }\end{array}$ & 11 & C & Parda & 5 & 3 & $\begin{array}{l}\text { Hipertensão grave/ } \\
\text { plaquetopenia/uso de } \\
\text { sulfato de magnésio }\end{array}$ \\
\hline Entrevistada 13 & 31 & Solteira & 12 & D & Preta & 5 & 0 & $\begin{array}{l}\text { Hipertensão arterial } \\
\text { grave }\end{array}$ \\
\hline Entrevistada 14 & 26 & $\begin{array}{l}\text { Mora } \\
\text { junto }\end{array}$ & 8 & C & Parda & 2 & 4 & $\begin{array}{l}\text { Hipertensão arterial } \\
\text { grave/pré-eclampsia } \\
\text { grave/uso de sulfato } \\
\text { de magnésio }\end{array}$ \\
\hline Entrevistada 15 & 26 & Solteira & 8 & D & Branca & 5 & 0 & $\begin{array}{l}\text { Descolamento } \\
\text { prematuro de } \\
\text { placenta }\end{array}$ \\
\hline Entrevistada 16 & 25 & $\begin{array}{l}\text { Mora } \\
\text { junto }\end{array}$ & 12 & C & Parda & $\begin{array}{c}\text { Sem } \\
\text { informação }\end{array}$ & 2 & $\begin{array}{l}\text { Hipertensão arterial } \\
\text { grave }\end{array}$ \\
\hline
\end{tabular}

Síndrome HELLP: hemolysis elevated liver enzymes low platelets.

* De acordo com o Critério de Classificação Econômica Brasil 20.

No presente estudo, a decisão pela busca do serviço de saúde também partiu em muitos casos da mulher, e em outras situações a mãe e o marido tiveram a iniciativa, evidenciando a importância do subsistema popular na tomada de decisão para procura por cuidados. Nesse subsistema as pessoas que mais se envolvem na tomada de decisão sobre o cuidado são familiares, amigos e vizinhos 25 . $\mathrm{O}$ apoio e a segurança que podem decorrer de uma união estável, por exemplo, contribuem para um maior cuidado das mulheres em relação à sua saúde 26 . 
Nem todas as entrevistadas utilizaram o primeiro nível do sistema de saúde ao adoecerem, visto que algumas procuraram as unidades de pronto atendimento (UPA), emergências de hospitais e outras se dirigiram diretamente para o nível terciário (hospital de referência). Os serviços de saúde brasileiros são organizados em níveis de complexidade; o acesso dos usuários aos diferentes níveis de cuidado deve ocorrer de modo que os quadros mais ou menos graves tenham acesso a instituições com adequada capacidade de resolução dos problemas de saúde.

$\mathrm{Na}$ gravidez, a mulher pode desenvolver complicações graves e os integrantes do subsistema popular reconhecem esse risco ao levá-la diretamente para os serviços de emergência e não para a atenção primária cujo aparato técnico-científico não é direcionado para quadros graves.

"É que não tem muito que fazer no posto de saúde [unidade básica de saúde - UBS], era só as consultas de rotina, então nunca procurei o posto por eu tá passando mal. Quando eu passei mal procurei o [nome do hospital]" (Entrevistada 5).

“...porque lá [UBS] eu fazia o exame hoje e eu ía saber o resultado daí a um mês, quando eu voltava na consulta, tanto que as duas vezes que eu tive infecção de urina durante a gravidez eu descobri na UPA, porque eu fazia exame no posto e demorava um mês pra ficar pronto, quando eu voltava eu já tinha ido na UPA porque eu sentia dor, ía na UPA e o diagnóstico é no mesmo dia por ser pronto atendimento" (Entrevistada 8).

Nos casos acima relatados, as mulheres foram encaminhadas para dar continuidade ao pré-natal no hospital de referência por se tratar de gestação de alto risco, as dificuldades referidas ocorreram enquanto realizavam o pré-natal na atenção básica, mas nas entrevistas elas deixam claro que os problemas relacionados à assistência foram resolvidos quando passaram a ser acompanhadas no ambulatório do hospital terciário.

As entrevistadas que realizaram o pré-natal no hospital de referência procuraram este local ao apresentarem sintomatologia. Isso ocorre pelo fato de as mulheres que fazem acompanhamento de pré-natal de alto risco na instituição ser orientadas pelos profissionais a procurar o serviço de referência caso apresentem sintomas.

Por conta do maior risco de complicações no ciclo gravídico-puerperal e a importância de atendimento ágil e resolutivo para a prevenção do óbito, torna-se imprescindível que os serviços de urgência e emergência ofereçam atendimento 24 horas para essas mulheres, sem a necessidade de fluxos de encaminhamento de outras instituições.

Segundo a antropologia médica, para que o usuário busque auxílio terapêutico, é necessário que ele reconheça ter um problema de saúde 15 . Contudo, para algumas mulheres, esse reconhecimento pode ocorrer de maneira equivocada, sem noção da real gravidade, como pôde ser constatado no presente estudo, quando duas mulheres não procuraram o serviço de saúde ao adoecerem e optaram pela automedicação.

"Oh, ensinaram um monte de chá pra minha mãe sabe, chá de matruz, não sei como que é o nome, alguns chás de pau que eu tomei pra aliviar a dor, acho que foi só isso, só que o nome eu não sei” (Entrevistada 9).

“...eu aumentei a dose por conta própria, que eu tava tomando de duzentas e cinquenta e comecei a tomar de quinhentas. (...) eu aumentei porque (...) porque eu vi que num tava baixando, entendeu, no momento que eu vi que num tava baixando eu aumentei..." (Entrevistada 12).

A prática da automedicação pode ser desastrosa e estar associada a determinados grupos sociais, à dificuldade de acesso ao serviço de saúde, cuja oferta ainda é distribuída desigualmente para as diferentes classes sociais, à ampla disponibilidade de medicações 16,27, à percepção equivocada por usuária e familiares da não gravidade do quadro e à liberdade de escolha influenciada pela subjetividade e aspectos socioculturais de cada mulher.

\section{Dificuldades de acesso aos serviços de saúde}

Na maioria dos casos, as mulheres foram encaminhadas em curto espaço de tempo (uma a duas horas), diretamente das unidades de saúde onde receberam o primeiro atendimento para o hospital de referência, mas a peregrinação por serviços de saúde e a demora do encaminhamento para o hospital foi vivenciada por outras entrevistadas.

"Então, eu fui no posto [UBS] aí as menina falou assim oh seu médico não tá aqui, vai na Santa Casa, aí quando eu cheguei na Santa Casa eles nem quiseram olhar, porque eles falou que se fosse erro do hospital [referência] eles não ía se responsabilizar, ai falou que eu ia ter que vim pra cá. Aí eu fui pra um postinho que fica 
lá perto da minha casa, aí chegou lá no postinho o enfermeiro examinou, aí a médica também me examinou, ela pegou e me encaminhou pro UPA, ai do UPA que me encaminhou pra cá..." (Entrevistada 9).

A Região de Saúde onde se localiza o hospital de referência possui fluxos de encaminhamentos formalmente estabelecidos para as situações de morbidade materna grave, e todos esses casos devem ser encaminhados para o hospital terciário onde a pesquisa foi realizada, mas o relato acima mostra que ainda há problemas em relação aos fluxos de atendimento nos serviços de atenção primária e secundária.

As mulheres que já estavam vinculadas ao hospital de referência, devido à realização do pré-natal na instituição, não encontraram dificuldades de atendimento no momento do adoecimento, já que se dirigiam diretamente ao hospital e recebiam atendimento imediato.

A ausência de acolhimento em diversos serviços pôde ser encontrada no relato acima, quando as demandas da entrevistada não foram atendidas, predominando a ausência de resolutividade e de compromisso com a usuária que necessita de atendimento. Alguns profissionais de saúde não realizam o acolhimento, desenvolvendo diversas outras tarefas de maneira simultânea e tecnicista, sem reflexão sobre aquilo que faz 13 .

O acolhimento envolve compromisso com o usuário, escuta e atendimento das demandas do indivíduo, e a assistência deve ser realizada de maneira ética, acolhendo o indivíduo em suas dores 28 .

A burocratização do atendimento também é um importante aspecto que dificulta o acesso aos serviços de saúde, uma vez que as normas e condutas passam a ser supervalorizadas em detrimento das reais necessidades das usuárias.

Em pesquisa no Rio de Janeiro, observou-se que o percurso das gestantes com síndromes hipertensivas entre a UBS e o hospital de referência nem sempre era direto, havendo situações de peregrinação na busca por assistência 13 .

Estudo multicêntrico, nacional, identificou que entraves no encaminhamento e transferência das mulheres com complicações graves consistem nos principais problemas para a adequada assistência obstétrica de emergência, o que acontece, sobretudo, nas transferências do nível secundário para o terciário 24.

Deficiências na assistência obstétrica também foram encontradas em maternidades do Nordeste brasileiro, em pesquisa que avaliou a estrutura das instituições por meio de itens como recursos humanos e materiais, planta física, materiais de consumo, ferramentas de gestão e educação em saúde. Nenhuma instituição atingiu nível de estrutura suficiente (sete foram classificadas como nível intermediário e duas como insuficientes) 29.

O presente estudo revelou dificuldades na organização e gestão dos serviços, em particular na logística da assistência e na garantia de atendimento em tempo hábil, sendo esses problemas enfrentados por algumas mulheres residentes em municípios vizinhos daquele onde está o hospital de referência, o que indica a distância como um importante aspecto para o acesso ao serviço terciário. Houve caso de espera de até oito horas para o encaminhamento de complicação grave, por dificuldades da gestão municipal em viabilizar transporte adequado.

"A hora que eu cheguei já começaram a ver vaga, mas aí eu tive que esperar o SAMU [Serviço de Atendimento Móvel de Urgência] daqui pra ir me buscar lá, porque o SAMU de lá [outro município] não tinha UTI, aí conforme o negócio de vaga para eu ter o SAMU disponível, com UTI e tudo (...). Das nove e meia, eles chegaram lá umas quatro e meia" (Entrevistada 1).

No relato acima, encontram-se importantes fragilidades nos arranjos regionais para a garantia da atenção integral, por isso são necessárias análises mais específicas sobre o funcionamento da Região de Saúde em relação aos entraves para o acesso ao serviço terciário em tempo hábil e de modo resolutivo.

É importante destacar que, se uma mulher com complicação grave demora em chegar ao hospital, a probabilidade de morrer aumenta, mesmo que a instituição de destino seja de nível terciário, com equipamento adequado e equipe qualificada 6 .

As dificuldades das mulheres na busca por cuidados durante a gestação mostram que a concretização da assistência integral está longe de ser efetivada, e as distintas necessidades apresentadas por essas mulheres, muitas vezes não são consideradas pelo profissional de saúde 13,30.

Entre as usuárias que fizeram pré-natal no hospital de referência, aconteceram situações de necessidade de hospitalização por complicação constatada durante realização de exames de rotina. "...quando foi na terça-feira eu vim fazer o perfil, cheguei aqui [hospital terciário] oito horas, ai quando foi de tardezinha que 
eu tive todos os exames, minha pressão tava alta ai eles falaram olha a gente vai ter que te internar que se você for pra casa não vai dar tempo de você voltar aqui de novo, por que você é de [outro município]..." (Entrevistada 3).

Nem sempre as mulheres apresentaram sintomas que sinalizassem a complicação, o que pôde ser constatado durante acompanhamento no serviço de saúde, verificando-se em tais casos o início do itinerário terapêutico da morbidade materna grave pelo subsistema profissional.

Pesquisa realizada em Ribeirão Preto, São Paulo, verificou que a hospitalização durante o ciclo gravídico-puerperal foi vista como um aspecto negativo pela mulher, podendo significar a concretização da gravidade do quadro e preocupação com o cuidado dos filhos e dos demais familiares que permaneceram no domicílio 31 . Todavia, os benefícios do internamento dos quadros graves são imensuráveis em relação à possibilidade de interrupção da evolução do adoecimento e prevenção do óbito, ainda que possa representar experiência traumática para as mulheres e familiares.

No presente estudo, a análise das dificuldades que as usuárias encontraram ao procurar assistência por causa do adoecimento evidencia que importantes barreiras como falta de acolhimento, demora no atendimento ou na constatação da necessidade de encaminhamento ao serviço de referência ocorreram, sobretudo, nas UPA e Santas Casas dos municípios vizinhos.

\section{Qualidade da assistência}

Metade das entrevistadas afirmou que os seus atendimentos foram adequados, a outra metade relatou problemas nos serviços por onde passaram, mas nenhuma apontou dificuldades no hospital de referência. Foram citados entraves desde a realização do pré-natal até os atendimentos não resolutivos na complicação.

"Eu acho assim, lá, principalmente no posto [UBS] (...), o atendimento não é legal, se eles te pede um exame demora um mês pra ficar pronto, a ginecologista, (...) não sei se é professora o que que ela é lá, é muito difícil o dia que você vai pra consulta que o estudante te atende e ela também vem pra conversar com você. Geralmente é o estudante que já vem com a receita pronta, te pede um monte de exame e quando você volta já é outro estudante, aí você que tem que falar pra outra pessoa o quê que tá acontecendo, repassar tudo" (Entrevistada 8).

“...eu não conseguia controlar a pressão à noite, ela subia mais à noite, eu ía na UPA lá de [outro município], eles me davam Nifedipina e eu ía pra casa, ela baixava e eu vinha pra casa, aí eu ficava controlando em casa, foi cinco dias isso, até o dia da consulta aqui no [hospital de referência]. (...) chegou um dia que eu fui na UPA e pedi pra eles me encaminharem pra cá, ai a médica de lá falou que não podia, me deu o remédio e mandou eu voltar pra casa" (Entrevistada 12).

Relatos de morbidade materna grave relacionados às complicações hipertensivas foram frequentes neste estudo, assim como em outra pesquisa realizada em Ribeirão Preto, o que torna patente a necessidade de um melhor rastreio e acompanhamento à hipertensão durante o pré-natal 31.

Estudo em hospitais brasileiros identificou que 25,7\% dos casos de morbidade materna grave e óbito materno apresentaram problemas assistenciais, como falta de equipamentos e medicamentos, não qualificação da equipe de saúde e demora para o diagnóstico 24 .

Observa-se no relato acima a ausência de organização da equipe de saúde, fato que dificulta a estruturação de um projeto terapêutico centrado na mulher que priorize o cuidado. A assistência à saúde deve ir além do tecnicismo, permitir a interação entre os sujeitos envolvidos no processo de cuidar e a compreensão das diversas singularidades 32 .

Os sistemas de saúde são formados por interações que envolvem aspectos culturais, relações de poder, normas que determinam tratamentos e relações interinstitucionais, por isso os estudos sobre sistemas de saúde não devem estar centrados apenas na terapêutica 19, mas também nos determinantes de saúde do adoecimento a exemplo das especificidades de gênero, nas quais as mulheres em muitas situações são duplamente vitimadas porque estão na situação de pacientes e de pessoas do sexo feminino 33 .

No presente estudo, foram relatados casos de violência institucional no momento do atendimento à morbidade materna grave:

“...todos os dias me tratavam mal lá [UPA]. (...) eles demoravam o atendimento pra mim e na hora que eu ía consultar eles fala oh você pode ficar de repouso, mas só que eles colocavam na cadeira, repouso que eu sei pra gestante é deitar do lado esquerdo, então eles me deixava na cadeira e era um descaso só, então eu ía reclamar, eu começava a chorar (...) ai elas me tratava mal por causa disso, porque eu chorava..." (Entrevistada 12). 
"Aí acho que eles têm que ficar mais esperto né, os médico também, os enfermeiro, porque tem uns que é grosso né, ignorante, você pergunta as coisa eles vêm com ignorância te responder, às vezes nem te responde [UBS e Santa Casa do município]” (Entrevistada 9).

As situações de violência institucional narrados ocorreram nas UBS, UPA e Santas Casas, e nenhum caso foi relatado no hospital de referência.

Relações hierárquicas desiguais entre profissional de saúde e usuário, baseadas no autoritarismo, podem se manifestar em agressões verbais, recusa em fornecer informações e demora do atendimento, caracterizando a violência institucional 28 .

Em pesquisa realizada em maternidades de São Paulo, maus-tratos e desrespeito foram reconhecidos por muitos dos profissionais como presentes em suas práticas, e alguns identificaram atos como violência, mas para outros tais práticas são necessárias para o "comportamento inadequado" das mulheres quando choram, gritam ou estão irritadas com o atendimento 33 .

Estudo analisou as narrativas de mulheres que desenvolveram complicações maternas graves, residentes em diferentes estados brasileiros, e mostrou relatos de agressões verbais e realização de procedimentos não recomendados como a manobra de Kristeller 14.

Vivências traumáticas como a violência institucional podem afetar a busca futura das mulheres por assistência, dificultar o estabelecimento de vínculos e a aceitação por procedimentos e orientações oferecidos pelos profissionais.

Todas as entrevistadas relataram ausência de dificuldades no hospital de referência. Entretanto, deve-se destacar que a satisfação também pode ocorrer pelo fato de a instituição ter recursos tecnológicos mais sofisticados, sendo considerado em razão disso um lugar de melhor atendimento 13. A avaliação de um bom atendimento também pode ser mascarada por questões relacionadas à responsividade, que inclui o baixo senso crítico da clientela do serviço de saúde devido à falta de conhecimento sobre o atendimento e procedimentos, além da possível relação de dependência estabelecida entre usuários e profissionais 34 .

\section{Perda de seguimento de casos de morbidade materna grave}

Embora o presente estudo não tenha pesquisado a perda de seguimento de casos de morbidade materna grave no pós-parto, situações como essa fazem parte de uma falha de acesso ao serviço de saúde. Aconteceu um caso que mostrou que a falta de acompanhamento adequado no puerpério pode levar a morbidade materna grave com a capacidade de afetar a qualidade de vida da mulher acometida.

"Então num foi de agora, já tinha desde a outra gravidez, aí ela ficou estabilizada, aí depois que eu ganhei nenê eu não voltei no médico aí a pressão começou a subir, subir, subir e eu não sentia nada, aí quando foi ver já tava tarde, aí eu já fui direto pra hemodiálise, eu perdi os rins por causa da pressão (...) aí dessa vez a pressão alterou muito nessa gravidez..." (Entrevistada 16).

Muitas mulheres que apresentaram complicações na gestação ou parto não percebem que ainda no pós-parto podem estar em risco, e também não são orientadas sobre a importância do acompanhamento clínico no puerpério, sendo priorizada a saúde do bebê 13 .

O Ministério da Saúde preconiza a realização de ao menos uma consulta puerperal, até o $42^{\circ}$ dia de pós-parto 35 . Todavia, a depender da gravidade da complicação no ciclo gravídico-puerperal, esse acompanhamento deve ser estendido para além de 42 dias de puerpério.

O acompanhamento pós-aborto também é essencial, especialmente ao se analisarem as frequentes complicações decorrentes de abortos inseguros. Porém, pesquisa realizada no Nordeste brasileiro com mulheres hospitalizadas em situação de aborto indica que apenas 4,3\% das entrevistadas nos hospitais de São Luís, Maranhão, tiveram a consulta de revisão pós-alta agendada 36.

\section{Conclusão}

Os itinerários terapêuticos percorridos por mulheres com morbidade materna grave são muito diversos e incluem desde a atenção básica até hospitais de referência. Existiram relatos de busca por recursos 
informais como a vizinha, além do uso de remédios populares como chás. Destacam-se também as interações familiares na iniciativa da busca do cuidado.

Alguns pontos críticos foram verificados no estudo em relação à assistência obstétrica, a exemplo da peregrinação por diversos serviços de saúde no momento da complicação, demora do encaminhamento do serviço de atendimento inicial para o hospital de referência, ausência de acolhimento às demandas das mulheres e violência institucional.

Identificou-se adequado funcionamento do hospital de referência no atendimento às morbidades maternas graves, tanto para as mulheres que já realizavam o pré-natal na instituição, por tratar-se de uma gestação de alto risco, quanto para aquelas encaminhadas de outros serviços de saúde.

Uma limitação do estudo foi as mulheres terem sido entrevistadas na instituição onde estavam hospitalizadas, e isso pode ter influenciado para que ocultassem possíveis entraves naquele hospital.

Com base neste estudo, novas pesquisas nas demais regiões do Estado de São Paulo e de outros estados podem ser desenvolvidas para a compreensão das diversas realidades em que as mulheres vítimas de complicações maternas estão inseridas e esses resultados poderão explicar a associação entre morbidade materna grave e a utilização de serviços de saúde, de acordo com as especificidades regionais.

\section{Colaboradores}

M. S. Andrade e E. M. Vieira participaram da concepção e projeto, análise e interpretação dos dados, redação do artigo e revisão crítica relevante do conteúdo intelectual e aprovação final da versão a ser publicada. Ambas são responsáveis por todos os aspectos do trabalho na garantia da exatidão e integridade de qualquer parte da obra.

\section{Agradecimentos}

À Universidade de São Paulo.

\section{Referências}

1. World Health Organization; World Bank; United Nations Children's Fund; United Nations Population Fund. Trends in maternal mortality: 1990 to 2010 . Geneva: World Health Organization; 2012.

2. United Nations. Millennium Development Goals and beyond 2015. New York: United Nations; 2013.

3. Alkeman L, Chou D, Hogan D, Zhang S, Moler A, Gemmill A, et al. Global, regional, and national levels and trends in maternal mortality between 1990 and 2015, with scenario-based projections to 2030: a systematic analysis by the UN Maternal Mortality Estimation InterAgency Group. Lancet 2016; 387:462-74. 
4. United Nations Human Rights. Maternal mortality and morbidity and human rights. Geneva: United Nations Human Rights; 2013.

5. Dias MAB, Domingues RMSM, Schilithz AOC, Nakamura-Pereira M, Diniz CSG, Brum $\mathrm{IR}$, et al. Incidência do near miss materno no parto e pós-parto hospitalar: dados da pesquisa Nascer no Brasil. Cad Saúde Pública 2014; 30 Suppl 1:S169-81.

6. Haddad SM, Cecatti JC, Souza JP, Sousa MH, Parpinelli MA, Costa ML, et al. Applying the maternal near miss approach for the evaluation of quality of obstetric care: a worked example from a multicenter surveillance study. Biomed Res Int 2014; 2014:989815.

7. Say L, Souza JP, Pattinson RC. Maternal near miss - towards a standard tool for monitoring quality of maternal health care. Best Pract Res Clin Obstet Gynaecol 2009; 23:287-96.

8. Cabral ALLV, Martinez-Hemáez A, Andrade EIG, Cherchiglia ML. Itinerários terapêuticos: o estado da arte da produção científica no Brasil. Ciênc Saúde Coletiva 2011; 16:4433-42.

9. Thaddeus S, Maine D. Too far to walk: maternal mortality in context. Soc Sci Med 1994; 38:1091-100.

10. Norhayati MN, Surianti S, Hazlina NHN. Metasynthesis: experiences of women with severe maternal morbidity and their perception of the quality of health care. PLoS One 2015; 10:e0130452.

11. Santana DS, Cecatti JG, Surita FG, Silveira C, Costa ML, Souza JP, et al. Twin pregnancy and severe maternal outcomes: the world health organization multicountry survey on maternal and newborn health. Obstet Gynecol 2016; 127:631-41.

12. Zanette E, Parpinelli MA, Surita FG, Costa ML, Haddad SM, Sousa MH, et al. Maternal near miss and death among women with severe hypertensive disorders: a Brazilian multicenter surveillance study. Reprod Health 2014; 11:4.

13. Xavier RB, Bonan C, Silva KS, Nakano AR. Healthcare itineraries of women with histories of hypertensive syndromes during pregnancy. Interface (Botucatu, Online) 2015; 19:1109-20.

14. Aguiar CA, Tanaka ACA. Memórias coletivas de mulheres que vivenciaram o near miss materno: necessidades de saúde e direitos humanos. Cad Saúde Pública 2016; 32:e00161215.

15. Kleinman A. Concepts and a model for the comparison of medical system as cultural system. Soc Sci Med 1978; 12:85-93.

16. Gerhardt TE. Itinerários terapêuticos em situações de pobreza: diversidade e pluralidade. Cad Saúde Pública 2006; 22:2449-63.

17. Alves PCB, Souza IM. Escolha e avaliação de tratamento para problemas de saúde: considerações sobre o itinerário terapêutico. In: Rabelo MC, Alves PCB, Souza IMA, organizadores. Experiência de doença e narrativa. Rio de Janeiro: Editora Fiocruz; 1999. p. 133-6.

18. Marconi MA, Lakatos EM. Fundamentos de metodologia científica. São Paulo: Editora Atlas; 2003.
19. Gomes R. Análise e interpretação de dados de pesquisa qualitativa. In: Minayo MCS, organizadora. Pesquisa social: teoria, método e criatividade. Rio de Janeiro: Editora Vozes; 2010. p. 79-108.

20. Associação Brasileira de Empresas de Pesquisa. Critério de classificação econômica Brasil. São Paulo: Associação Brasileira de Empresas de Pesquisa; 2015.

21. Godoy SR, Gualda DMR, Bergamasco RB, Tsunechiro MA. Percepção da causalidade e dos sinais de near miss atribuídos por mulheres sobreviventes. Texto Contexto Enferm 2008; 17:536-43.

22. Pinho CAP, Pamplona TVL, Lima PCMG. Feelings and perceptions of women in the pregnancy-puerperal cycle who survived severe maternal morbidity. Rev Latinoam Enferm 2010; 18:1187-94.

23. Kleinman A. Patients and healers in the context of culture. Berkeley: University of California Press; 1980.

24. Pacagnella RC, Cecatti JG, Parpinelli MA, Sousa MH, Haddad SM, Costa ML, et al. Delays in receiving obstetric care and poor maternal outcomes: results from a national multicentre cross-sectional study. BMC Pregnancy Childbirth 2014; 14:159.

25. Mattosinho MMS, Silva DMGV. Therapeutic itinerary of the family and adolescent with type I mellitus diabetes. Rev Latinoam Enferm 2007; 15:1113-9.

26. Peretto M, Lopes MJM, Soares JSF, Swarowsky GE. Gravidez na adolescência em oito municípios do RS: perfil de ocorrência e rede de serviços. Rev Eletrônica Enferm 2011; 13:721-9.

27. Mendes EV. As redes de atenção à saúde. Brasília: Organização Pan-Americana da Saúde; 2011.

28. Ministério da Saúde. Acolhimento nas práticas de produção de saúde. Brasília: Ministério da Saúde; 2010.

29. Alves MTSSB, Araújo TVB, Alves SV, Marinho LFB, Schiavo E, Menezes G, et al. Atenção ao aborto no Sistema Único de Saúde no Nordeste Brasileiro: a estrutura dos serviços. Rev Bras Saúde Matern Infant 2014; 14:229-39.

30. Cabrita BAC, Abrahão AL, Rosa AP, Rosa FSF. A busca do cuidado pela gestante de alto risco e a relação integralidade em saúde. Ciênc Cuid Saúde 2015; 14:1139-48.

31. Silva DVR, Silveira MFA, Sponholz FAG. Experiências em morbidade maternal grave: estudo qualitativo sobre a percepção de mulheres. Rev Bras Enferm 2016; 69:662-8.

32. Silva NEK, Sancho LG, Figueiredo WS. Entre fluxos e projetos terapêuticos: revisitando as noções de linha do cuidado em saúde e itinerários terapêuticos. Ciênc Saúde Coletiva 2016; 21:843-52. 
33. Aguiar JM, d'Oliveira AFPL, Schraiber LB. Violência institucional, autoridade médica e poder nas maternidades sob a ótica dos profissionais de saúde. Cad Saúde Pública 2013; 29:2287-96

34. Vaitsman J, Andrade GRB. Satisfação e responsividade: formas de medir a qualidade e a humanização da assistência à saúde. Ciênc Saúde Coletiva 2005; 10:599-613.
35. Ministério da Saúde. Atenção ao pré-natal de baixo risco. Brasília: Ministério da Saúde; 2012.

36. Aquino EML, Menezes G, Barreto-de-Araújo TV, Alves MT, Alves SV, Almeida MCC, et al. Qualidade da atenção ao aborto no Sistema Único de Saúde do Nordeste brasileiro: o que dizem as mulheres? Ciênc Saúde Coletiva 2012; 17:1765-76.

\section{Abstract}

This study aimed to identify the treatment itineraries of Brazilian women with severe maternal morbidity. This was a qualitative, descriptive, exploratory study in a university referral hospital for high-risk pregnancy in the interior of São Paulo State, Brazil. The sample included 16 women with severe complications during pregnancy, childbirth, or postpartum. Data were collected with semi-structured, taped, transcribed interviews subsequently submitted to Content Analysis. The results showed that at the onset of illness, the women take various paths to reach low and highcomplexity services, while also relying on neighbor women, pharmacies, and self-medication. They encounter obstacles in the services' receptiveness, continuity of care, case-resolution capacity, and referrals within the healthcare network. Critical points were observed in obstetric care: women are frequently subjected to trekking from one health service to another to obtain care, delays in referrals, and institutional violence. The tertiary hospital was described as receptive and effective. The study identified problems in the entire chain of obstetric care, helping explain how the healthcare system is organized to deal with severe maternal complications.

Morbidity; Women's Health; Comprehensive Health Care

\section{Resumen}

Este estudio tuvo por objetivo identificar los itinerarios terapéuticos de mujeres afectadas por morbilidad materna grave. Se trata de una investigación cualitativa, descriptiva y exploratoria, realizada en un hospital universitario de referencia en gestación de alto riesgo del interior del Estado de São Paulo, Brasil. Participaron 16 mujeres con complicaciones graves durante la gestación, parto o posparto. Los datos fueron recogidos gracias a entrevistas semiestructuradas, grabadas, transcritas y analizadas mediante Análisis de Contenido. Los resultados muestran que las mujeres, al comenzar el proceso de padecimiento, siguen trayectorias diversas, buscando servicios de baja y alta complejidad, recurriendo también a vecinas, farmacias y a la automedicación. Se destacan trabas en relación con la acogida, continuidad del cuidado, resolutividad y referentes a la red de atención a la salud. Se observaron importantes puntos críticos, respecto a la asistencia obstétrica, siendo la peregrinación por servicios de salud, la tardanza en la derivación a especialistas y la violencia institucional, realidades vividas por las mujeres. El servicio terciario fue descrito como acogedor y eficaz en la atención. Este estudio permitió la identificación de los problemas que existen en toda la cadena de cuidados obstétricos, y este conocimiento permite el entendimiento de cómo el sistema de salud se organiza en relación a la atención de las mujeres con complicaciones maternas graves.

Morbidad; Salud de la Mujer; Atención Integral de la Salud

Recebido em 30/Mai/2017

Versão final reapresentada em 20/Nov/2017

Aprovado em 26/Dez/2017 